\title{
Plasma N-terminal propeptide of type III procollagen accurately predicts liver fibrosis severity in children with non-alcoholic fatty liver disease
}

Running title: PIIINP for predicting liver fibrosis

Antonella Mosca ${ }^{1}$, Donatella Comparcola ${ }^{2}$, Ilaria Romito ${ }^{3}$, Alessandro Mantovani ${ }^{4}$, Valerio Nobili $^{1,5^{*}}$, Christopher D. Byrne ${ }^{6,7}$, Anna Alisi ${ }^{3 \#}$, Giovanni Targher ${ }^{4 \#}$

${ }^{*}$ Deceased

\# These two authors contributed equally to the manuscript.

${ }^{1}$ Hepatology Gastroenterology and Nutrition, Bambino Gesù Children's Hospital, Rome, Italy

${ }^{2}$ Hepato-Metabolic Disease Unit, Bambino Gesù Children's Hospital, IRCCS, Rome, Italy

${ }^{3}$ Research Unit of Molecular Genetics of Complex Phenotypes, Bambino Gesù Children's Hospital, IRCCS, Rome, Italy

${ }^{4}$ Section of Endocrinology, Diabetes and Metabolism, Department of Medicine, University and Azienda Ospedaliera Universitaria Integrata of Verona, Verona, Italy

${ }^{5}$ Department of Pediatrics, University La Sapienza, Rome, Italy

${ }^{6}$ Southampton National Institute for Health Research Biomedical Research Centre, University Hospital Southampton, Southampton General Hospital, Southampton, UK

${ }^{7}$ Nutrition and Metabolism, Faculty of Medicine, University of Southampton, UK

Word count: 248 abstract; 4200 text (excluding manuscript title, abstract, lay summary, references, tables and figure legends). Tables=4, Figures=4, Supplementary Figures=2

Keywords: children, liver fibrosis, NAFLD, PIIINP, procollagen 
Address Correspondence:

Dr. Anna Alisi, Research Unit of Molecular Genetics of Complex Phenotypes, Bambino Gesù Children's Hospital, IRCCS, Viale San Paolo 15, 00146 Rome, Italy. Phone: +39066859 2186. E-mail: anna.alisi@opbg.net

Prof. Giovanni Targher, MD, Section of Endocrinology, Diabetes and Metabolism Department of Medicine University and Azienda Ospedaliera Universitaria Integrata Piazzale Stefani, 1, 37126 Verona, Italy, Phone: +39-0458123110. E-mail: giovanni.targher@univr.it 


\section{LIST OF ABBREVIATIONS}

ALT: alanine aminotransferase

APRI: AST to platelet ratio index

AST: aspartate aminotransferase

AUROC: area under the receiver operating characteristic curve

BMI: body mass index

ECM: extracellular matrix

ELF: enhanced liver fibrosis

ELISA: enzyme-linked immunosorbent assay

FIB-4: fibrosis-4

GGT: gamma-glutamyltransferase

HDL: high-density lipoprotein

HOMA-IR: homeostasis model assessment-insulin resistance

LDL: low-density lipoprotein

NAFLD: non-alcoholic fatty liver disease

NAFL: non-alcoholic fatty liver

NAS: NAFLD Activity Score

NASH: non-alcoholic steatohepatitis

NPV: negative predictive values

PIIINP: N-terminal propeptide of type III procollagen

PNPLA3: patatin-like phospholipase domain-containing protein-3

PPV: positive predictive value

ROC: receiver operating characteristic curve

Financial Support: 
This study was supported by the Italian Ministry of Health funds (Fondi di Ricerca Corrente 2018) to Dr. Anna Alisi. CDB is supported in part by grants from the Southampton National Institute for Health Research Biomedical Research Centre. GT is supported in part by grants from the University School of Medicine of Verona, Verona, Italy.

Disclosure Statement: The Authors have no potential conflicts of interest to disclose.

\section{Author Contributions:}

Antonella Mosca: patients visit, data collection, interpretation of data, drafting of the manuscript, critical revision of the manuscript, and final submission approval. Donatella Comparcola: patients visit, data collection, critical revision of the manuscript, and final submission approval. Ilaria Romito: laboratory analyses, drafting of the manuscript, critical revision of the manuscript, and final submission approval. Alessandro Mantovani: statistical analyses, critical revision of the manuscript, and final submission approval. Valerio Nobili (deceased): study concept and design, patients visit, and data collection. Christopher D. Byrne: drafting of the manuscript, critical revision of the manuscript, and final submission approval. Anna Alisi: study concept and design, interpretation of data, drafting of the manuscript, critical revision of the manuscript, and final submission approval. Giovanni Targher: statistical analyses, drafting of the manuscript, critical revision of the manuscript, and final submission approval.

\section{Acknowledgements:}

We thank Dr. Rita De Vito for the evaluation of liver biopsies. 


\section{ABSTRACT}

Background \& Aims: We examined the diagnostic performance of plasma N-terminal propeptide of type III procollagen (PIIINP) levels, aspartate aminotransferase to platelet ratio index (APRI) and Fibrosis-4 (FIB-4) score for predicting nonalcoholic steatohepatitis (NASH) and liver fibrosis stage in children/adolescents with non-alcoholic fatty liver disease (NAFLD).

Methods: We enrolled 204 children/adolescents with biopsy-proven NAFLD at the "Bambino Gesù" Children's Hospital. We measured plasma PIIINP levels using a commercially available enzyme-linked immunosorbent assay kit and calculated APRI and FIB-4 scores using standard methods.

Results: Children with NASH had higher plasma PIIINP levels, APRI and FIB-4 scores compared with those without NASH (all $p<0.001$ ). However, PIIINP levels had much better diagnostic performance and accuracy than APRI and FIB-4 scores for predicting liver fibrosis stage. PIIINP levels correlated to the total NAFLD activity score (NAS) and its constituent components $(p<0.0001)$. The risk of either NASH or $\mathrm{F} \geq 2$ fibrosis progressively increased with increasing PIIINP levels $(p<0.0001)$, independently of age, sex, adiposity measures, insulin resistance, NAS score and the patatin-like phospholipase domain-containing protein-3 rs738409 polymorphism. For every $3.6 \mathrm{ng} / \mathrm{mL}$ increase in PIIINP levels, the likelihood of having $\mathrm{F} \geq 2$ fibrosis increased by $\sim 14$-fold (adjusted-odds ratio $14.1,95 \% \mathrm{Cl} 5.50-35.8$, $p<0.0001)$ after adjustment for the aforementioned risk factors. The area under the receiver operating characteristics curve was $0.921(95 \% \mathrm{Cl} 0.87-0.97)$ for $\mathrm{F} \geq 2$ fibrosis, and 0.993 (95\% Cl 0.98-1.0) for F3 fibrosis, respectively.

Conclusions: Unlike APRI and FIB-4 scores, plasma PIIINP levels are a promising, noninvasive biomarker for diagnosing liver fibrosis stage in children/adolescents with biopsyproven NAFLD. 


\section{LAY SUMMARY}

There is an unmet need for non-invasive biomarkers and imaging techniques for the diagnosis of advanced liver fibrosis in pediatric NAFLD. Plasma levels of N-terminal propeptide of type III procollagen are a promising, non-invasive biomarker for staging liver fibrosis in children/adolescents with biopsy diagnosed NAFLD. 


\section{INTRODUCTION}

The childhood obesity epidemic has become a serious public health problem in most industrialized countries, and is also increasing the burden of several chronic noncommunicable diseases, including nonalcoholic fatty liver disease (NAFLD). ${ }^{1}$ NAFLD is a metabolic liver disease that encompasses a spectrum of progressive pathologic conditions, ranging from nonalcoholic fatty liver (NAFL) to steatohepatitis (NASH), advanced fibrosis and cirrhosis. ${ }^{2}$ Pediatric NAFLD is becoming the leading cause of chronic liver disease worldwide, affecting up to $10-15 \%$ of children/adolescents in Western countries. $^{3,4}$

Within the spectrum of NAFLD it is important to distinguish patients with early disease (NAFL) from those at greater risk of developing advanced fibrosis and cirrhosis. ${ }^{5-9}$ Indeed, many studies have reported that in adults with NASH the severity of liver fibrosis is the strongest predictor of all-cause mortality and liver-related complications, such as liver failure often requiring liver transplantation. ${ }^{10}$ However, a major obstacle in identifying $\mathrm{NASH}$, or assessing the severity of liver fibrosis is the need to undertake a liver biopsy to establish a histological diagnosis. ${ }^{10,11}$ The invasiveness, associated morbidity and costs of performing a liver biopsy make it unsuitable as a screening tool for diagnosing NAFLD and staging liver fibrosis. Although liver biopsy is mandatory for diagnosing $\mathrm{NASH}$, this technique often requires a general anesthetic in children, adding additional costs and potential risks of morbidity. Thus, in the last decade biomarker research in NAFLD has focused on two major needs: a) establishing a diagnosis of $\mathrm{NASH}$; and b) detecting and quantifying the presence of liver fibrosis. ${ }^{6,7}$ Among the biomarkers, the enhanced liver fibrosis (ELF) test, which uses a combination of three direct biomarkers of fibrosis [i.e., $\mathrm{N}$ terminal propeptide of type III procollagen (PIIINP), hyaluronic acid and tissue inhibitor of metalloproteinase 1], has been validated in cohorts of adults and children with NAFLD. ${ }^{12,13}$ In a small study of about 100 children with biopsy-proven NAFLD, Nobili et al. reported 
that the ELF test predicted liver fibrosis stage with a high degree of sensitivity and specificity. ${ }^{13}$ However, the ELF test is expensive and not widely available in countries outside the UK. Amongst the components of the ELF test, PIIINP is a procollagen III cleavage product that can be measured in blood samples and used as a circulating biomarker of extracellular matrix (ECM) remodeling during liver fibrogenesis. ${ }^{14}$ PIIINP measurement is much less expensive than the ELF test and the commercially available enzyme-linked immunosorbent assay (ELISA) kits for PIIINP are now more easily available on all commercial laboratory auto-analyzers. Interestingly, plasma PIIINP levels have been validated in a study involving nearly 170 UK middle-aged patients with biopsy-proven NAFLD as one of the most efficient biomarker that may differentiate between patients with NAFL and those with NASH or advanced fibrosis. ${ }^{15}$ However, to the best of our knowledge, no studies in children/adolescents with biopsy-proven NAFLD have investigated the diagnostic performance of plasma PIIINP levels for predicting the severity of liver fibrosis and other histologic features of NAFLD. In 2016, a small case-control study, involving 55 obese Egyptian children with ultrasound-diagnosed NAFLD, reported that plasma PIIINP levels might serve as a marker of hepatic injury and steatosis. ${ }^{16}$ Therefore, in children/adolescents who had undergone a diagnostic liver biopsy, the primary objective of our study was to investigate the diagnostic performance of plasma PIIINP levels for assessing the severity of the histologic features of NAFLD, whereas secondary objective of the study was to compare the diagnostic performance of the PIIINP assay to that of other two non-invasive markers of fibrosis, i.e., the Fibrosis-4 (FIB-4) score and the aspartate aminotransferase to platelet ratio index (APRI) that are widely adopted by clinicians for predicting advanced liver fibrosis in adults with NAFLD.

\section{MATERIALS AND METHODS}




\section{Patients}

We enrolled 204 children/adolescents (154 boys; 5-17 years) with an ultrasonographic diagnosis of severe hepatic steatosis or persistently ( $\geq 6$ months) elevated serum aminotransferase levels, who were consecutively referred to the Hepato-Metabolic Department of "Bambino Gesù” Children's Hospital (Rome) between January 2015 and March 2018, and who accepted to undergo liver biopsy for diagnosing the severity of NAFLD. This clinical practice is in agreement with the diagnostic flow chart proposed by the European Society for Pediatric Gastroenterology, Hepatology, and Nutrition (ESPGHAN) Hepatology Committee. ${ }^{17}$ Most of these children were overweight or obese. All children were tested to exclude secondary causes of hepatic steatosis (i.e., viral hepatitis, autoimmune hepatitis, Wilson's disease, $\alpha$-1-antitrypsin deficiency, endocrinological, genetic and metabolic diseases, celiac disease, alcohol consumption, and use of drugs known to induce hepatic steatosis). All included children were Caucasians of Italian descent. The study was performed according to the rules of the Helsinki Declaration. The study protocol was approved by the ethics committee of the “Bambino Gesù" Children's Hospital. Written informed consent was obtained from the parents of each child.

\section{Anthropometric and biochemical measurements}

Body weight and height were measured with the patient wearing underwear. Body mass index (BMI) and waist circumference were calculated as previously described. ${ }^{18,19}$ Blood pressure was measured in the right arm using a standard sphygmomanometer; the average of three blood pressure values was reported. Elevated blood pressure was defined by systolic or diastolic blood pressure $>95^{\text {th }}$ percentile for age, height and sex. Venous blood samples were collected in the morning after an overnight fast of at least 8 hours. Serum liver enzymes [aspartate aminotransferase (AST), alanine aminotransferase 
[ALT] and gamma-glutamyltransferase (GGT)], lipids [total cholesterol, high-density lipoprotein (HDL)-cholesterol, low-density lipoprotein (LDL)-cholesterol and triglycerides], fasting glucose and insulin levels were measured in all patients by using standard laboratory procedures at the central Laboratory of the "Bambino Gesù" Children's Hospital. Homeostasis model assessment score was used for estimating insulin resistance; a cut-off value of HOMA-IR >2.5 was considered as an index of insulin resistance. ${ }^{20}$

\section{Liver histology}

Liver biopsies were performed using an automatic core biopsy 16 or 18-gauge needle under general anesthesia and ultrasound guidance. The characterized histological features of NAFLD were hepatic steatosis, portal and lobular inflammation, hepatocyte ballooning and fibrosis. A single experienced liver pathologist (De Vito R, mentioned in the acknowledgements) evaluated all liver biopsies. NASH was characterized according to the scoring system developed by the National Institutes of Health-sponsored NASH Clinical Research Network. ${ }^{21}$ Briefly, hepatic steatosis was graded on a four-point scale: $0=$ steatosis involving fewer than $5 \%$ of hepatocytes, $1=$ steatosis involving up to $33 \%$ of hepatocytes, $2=$ steatosis involving $33 \%$ to $66 \%$ of hepatocytes, and $3=$ steatosis involving more than $66 \%$ of hepatocytes. Lobular inflammation was graded on a four-point scale: $0=$ no foci, $1=$ fewer than two foci per $200 \times$ field, $2=$ two to four foci per $200 \times$ field, and $3=$ more than four foci per $200 \times$ field. Portal inflammation was graded on a four-point scale: $0=$ none, $1=$ mild, $2=$ moderate, and $3=$ severe. Hepatocyte ballooning was graded on a three-point scale: $0=$ no balloon cells, $1=$ few balloon cells and $2=$ many/prominent balloon cells. The stage of liver fibrosis was quantified using a five-point scale: $0=$ no fibrosis, 1=peri-sinusoidal or periportal fibrosis [(1a) mild, zone 3, perisinusoidal; (1b) moderate, zone 3, perisinusoidal; and (1c) portal/periportal], 2=peri-sinusoidal and portal/periportal fibrosis, 3=bridging fibrosis, and 4=cirrhosis. According to the NASH Clinical Research 
Network, we also calculated the NAFLD Activity Score (NAS). ${ }^{22}$ NAS is defined as the sum of the scores for steatosis (0-3), lobular inflammation (0-3), and ballooning (0-2); thus, ranging from 0 to 8 . Liver fibrosis is not included as a component of this activity score. Cases with NAS of 0 to 2 were considered not diagnostic of NASH. Cases with activity scores of 3 and 4 were diagnosed as borderline $\mathrm{NASH}$, whereas all cases with scores of $\geq 5$ were diagnosed as definite $\mathrm{NASH}^{23}$

Non-invasive liver fibrosis scores

The APRI was calculated as AST/ULN (upper limit of normal)/platelet count $\mathrm{x} 100$, whereas the FIB-4 score was calculated using the following equation: FIB-4= (age $\times$ AST)/[platelet count $x \sqrt{ } \mathrm{ALT}] .^{24,25}$

\section{PIIINP quantitative assay}

The detection of PIIINP was performed in plasma samples by using Human PIIINP enzymelinked immunosorbent assay (ELISA) kit, provided by MyBiosource.com (Catolog Number: MBS703383), according to the manufacturer's directions. The assay has high sensitivity and excellent specificity for detection of human PIIINP. Standards and samples (dilution 1:20) were pipetted into the wells, pre-coated with antibody specific for PIIINP. After removing any unbound substances, a biotin-conjugated antibody specific for PIIINP was added to the wells. After washing, avidin conjugated Horseradish Peroxidase was added to the wells and then, a substrate solution was added. The color developed in proportion to the amount of PIIINP bound in the initial step and the intensity of the color was measured by using an ELISA microplate spectrophotometer with Tecan's proven Magellan ${ }^{\mathrm{TM}}$ software. Both intra- and inter-assay coefficients of variation for PIIINP were less than $10 \%$. Detection range for the assay was from $0.125 \mathrm{ng} / \mathrm{mL}$ to $8 \mathrm{ng} / \mathrm{mL}$. 


\section{PNPLA3 genotyping}

Blood samples were collected and the genomic DNA was extracted by QIAamp Blood MiniKit (Qiagen, Hilden, Germany), according to the manufacturer's instructions. The rate of success in extracting DNA was $100 \%$ for each study group. The patatin-like phospholipase domaincontaining protein-3 (PNPLA3) rs738409 C>G SNP, encoding I148M, was genotyped with a 5'-nuclease TaqMan assay (Assay on Demand for rs738409, Applied Biosystems, Foster City, CA) by personnel unaware of the clinical status of the patients. Post-polymerase chain reaction allelic discrimination was carried out through the measurement of allele-specific fluorescence on the Opticon 2 detection system (MJ Research, Waltham, MA). Random samples were confirmed by direct genotyping (Applied Biosystems 3500 Genetic Analyzer), which provided concordant results in all cases; controls were included in all analyzed batches, and quality controls were used to verify the reproducibility of the results. Distribution of the genotype was in Hardy-Weinberg equilibrium and the call rate exceeded $99 \%$.

\section{Statistical analysis}

Due to the hypothesis-generating nature of the design of the study, a prior sample size calculation was not undertaken. Data are expressed as means $\pm S D$, medians (inter-quartile ranges, IQR) or frequencies. Standard descriptive statistics were used to compare the differences in clinical, biochemical and liver histology characteristics according to the presence or absence of histologically confirmed NASH. These tests included the Fisher's exact test or the chi-squared test for categorical variables, the unpaired Student's $t$ test for normally distributed continuous variables, and the Mann-Whitney $U$ test for non-normally distributed variables. The Kruskal-Wallis test was also used to perform comparisons between mean PIIINP levels in relation to all histologic features of NAFLD (hepatic steatosis, lobular/portal inflammation, ballooning degeneration, fibrosis and NAS score) followed by Bonferroni's multiple comparison tests. Finally, a binary logistic regression analysis was used 
to examine the association between plasma PIIINP levels (expressed for each SD increment) and either the presence/absence of definite NASH (i.e., defined as NAS score $\geq 5$ ) or the presence/absence of $\mathrm{F} \geq 2$ fibrosis on histology, as binary outcome(s). In these logistic regression analyses, we adjusted for age, sex, adiposity measures (BMl or waist circumference) and HOMA-IR score. To further examine the association between plasma PIIINP levels and the presence/absence of $\mathrm{F} \geq 2$ fibrosis, we also performed an additional multivariable logistic regression model where NAS score and PNPLA3 genotypes were also included among the covariates. Covariates included in all these multivariable regression models were selected based on their significance in univariable analyses, or based on their biological plausibility as potential confounders. Receiver operating characteristic (ROC) curves, plotting sensitivity against 1 -specificity, were also used to assess the diagnostic performances of both plasma PIIINP levels and other common non-invasive markers of fibrosis (i.e., FIB-4 and APRI scores) for discriminating both NASH and liver fibrosis stage. Areas under ROC curve (AUROC) with 95\% confidence intervals $(95 \% \mathrm{Cl})$ were calculated under non-parametric (distribution free) assumption. Optimal cut-off values were calculated to maximise sensitivity and specificity. For each cut-off, sensitivity, specificity, positive predictive value (PPV) and negative predictive value (NPV) were reported. The Harrell's C statistics was used to compare the diagnostic performances of plasma PIIINP levels, FIB-4 and APRI scores. A p-value $<0.05$ was considered statistically significant. Statistical analyses were performed using STATA software, version 14.2 (STATA, College Station, Texas, USA).

\section{RESULTS}

PIIINP levels are increased in children/adolescents with NASH

Table 1 summarizes the main demographic, clinical and biochemical characteristics of children/adolescents with biopsy-proven NAFLD, stratified by presence or absence of definite 
$\mathrm{NASH}$. Compared to those without NASH, children/adolescents with NASH were more likely to be insulin resistant (as reflected by higher fasting insulin concentrations and greater HOMA-IR score) and had higher values of serum liver enzymes, APRI and FIB-4 scores, and lower platelet count. No significant differences were found in age, sex, adiposity measures (BMI and waist circumference), blood pressure and plasma lipid profile. Notably, as shown both in Table 1 and in Figure 1, children/adolescents with definite NASH had markedly higher plasma PIIINP levels compared with either children/adolescents with borderline NASH and/or no NASH considered together (panel A), or children/adolescents with no NASH and borderline NASH considered separately (panel B).

PIIINP levels are associated with liver fibrosis stage and other histologic features of NAFLD

Table 2 summarizes the histologic features of NAFLD of children/adolescents, stratified by NASH status. As expected, children/adolescents with NASH had more advanced histologic features (hepatic steatosis, inflammation, ballooning degeneration and fibrosis) than those without NASH, and their NAS score was higher. Figure 2 shows plasma PIIINP levels in relation to the various histologic features of NAFLD and NAS score. Plasma PIIINP levels increased sharply with increasing severity of liver fibrosis, ballooning degeneration, inflammation (lobular and portal) and steatosis. In particular, the strength of the association between PIIINP levels and liver histology features was very strong for liver fibrosis. In fact, PIIINP levels were nearly 9-fold higher in children/adolescents with F2 fibrosis, and $\sim$ 13-fold higher in those with F3 fibrosis, respectively, compared to their counterparts with a fibrosis stage of zero. Very similar results were also found when we re-analyzed the levels of plasma PIIINP in relation to liver fibrosis stages by dividing fibrosis stage 1 in 3 sub-stages, according to the NASH-CRN classification (plasma PIIINP levels in F0: $1.28 \pm 0.5 \mathrm{ng} / \mathrm{ml}$; F1a: $4.24 \pm 1.9 \mathrm{ng} / \mathrm{ml} ; \mathrm{F} 1 \mathrm{~b}: 6.82 \pm 1.1 \mathrm{ng} / \mathrm{ml} ; \mathrm{F} 1 \mathrm{c}: 8.06 \pm 1.9 \mathrm{ng} / \mathrm{ml} ; \mathrm{F} 2: 10.26 \pm 2.5 \mathrm{ng} / \mathrm{ml} ; \mathrm{F} 3$ : $14.61 \pm 1.4 \mathrm{ng} / \mathrm{ml} ; p<0.0001$ for trend). 
Furthermore, as also shown in the figure, there was a strong, graded association between increasing PIIINP levels and the total NAS score, with plasma PIIINP levels that markedly increased in presence of NAS score of at least three or more.

Table 3 and Table 4 show the association between plasma PIIINP levels and risk of having either definite $\mathrm{NASH}$ or $\mathrm{F} \geq 2$ fibrosis, before and after adjustment for covariates and potential confounding factors. In univariable regression analysis (unadjusted model), elevated plasma PIIINP levels (expressed for each SD increment, i.e. $3.6 \mathrm{ng} / \mathrm{mL}$ ) were associated with a 2.7fold increased risk of NASH (Table 3; unadjusted-OR 2.75, 95\% Cl 1.92-3.94, $p<0.0001$ ). This association remained significant even after adjustment for age, sex, BMI and HOMA-IR (adjusted model). Similarly, and most interestingly, for each SD increase in PIIINP levels, the likelihood of having $\mathrm{F} \geq 2$ fibrosis increased by $\sim 15$-fold after adjustment for age, sex, BMI and HOMA-IR score (Table 4, model 1: adjusted-OR 14.5, 95\% Cl 6.24-33.8, $p<0.0001$ ). Further adjustment for NAS score and PNPLA3 genotypes did not weaken the significant association between PIIINP levels and risk of $\mathrm{F} \geq 2$ fibrosis (Table 4, model 2: adjusted-odds ratio 14.1, $95 \% \mathrm{Cl} 5.50-35.8, p<0.0001)$. Almost identical results were found when we adjusted for waist circumference instead of BMI (data not shown).

Performance of PIIINP levels for discriminating NASH and liver fibrosis stage

The AUROC for plasma PIIINP levels for predicting NASH was estimated to be $0.737(95 \%$ Cl 0.66-0.81) (Figure 3, panel A). A cut-off of plasma PIIINP $>7.60 \mathrm{ng} / \mathrm{mL}$ (i.e., the optimum threshold) gave a sensitivity $62 \%$, specificity $91 \%$, PPV $85 \%$ and NPV $75 \%$ for discriminating definite NASH from no-NASH/borderline NASH. Importantly, there was excellent performance of PIIINP levels for predicting advanced stages of liver fibrosis. Indeed, AUROCs for PIIINP levels in predicting either $\mathrm{F} \geq 2$ fibrosis or $\mathrm{F} 3$ fibrosis were $0.921(95 \% \mathrm{Cl}$ 0.87-0.97) (panel B) and 0.993 (95\% Cl 0.98-1.0) (panel C), respectively. A cut-off of plasma PIIINP concentration of $>8.89 \mathrm{ng} / \mathrm{mL}$ yielded $84 \%$ sensitivity, $94 \%$ specificity, $95 \%$ PPV and 
79\% NPV for predicting $\mathrm{F} \geq 2$ fibrosis, whereas a cut-off of plasma PIIINP $>13.2 \mathrm{ng} / \mathrm{mL}$ yielded $100 \%$ sensitivity, $98 \%$ specificity, $70 \%$ PPV and $100 \%$ NPV for predicting the presence of $\mathrm{F} 3$ fibrosis (Figure 3).

Comparison of diagnostic performances of PIIINP levels, APRI and FIB-4 scores for discriminating $\mathrm{NASH}$ and fibrosis stage

Figure 4 shows that the diagnostic performance of PIIINP levels was significantly better than that of both FIB-4 and APRI scores in predicting definite NASH, F $\geq 2$ fibrosis or F3 fibrosis, respectively. Compared to plasma PIIINP levels, both scores had poor performance, especially a very low NPV, for predicting NASH and liver fibrosis stage. Supplementary Figures 1 and 2 show the AUROCs for both FIB-4 and APRI scores in predicting definite $\mathrm{NASH}, \mathrm{F} \geq 2$ fibrosis or F3 fibrosis, respectively.

\section{DISCUSSION}

In our mono-ethnic cohort of children/adolescents with biopsy-confirmed NAFLD, there are key novel findings. Compared to plasma PIIINP levels, both FIB-4 and APRI scores showed much poorer performance for predicting the more advanced stages of liver fibrosis. Plasma PIIINP levels showed excellent diagnostic performance for predicting the more advanced stages of liver fibrosis [i.e., AUROCs for plasma PIIINP levels for predicting either F $\geq 2$ fibrosis or $\mathrm{F} \geq 3$ fibrosis were $0.921(95 \% \mathrm{Cl} 0.87-0.97)$ and $0.993(95 \% \mathrm{Cl} 0.98-1.0)$, respectively]. For every $3.6 \mathrm{ng} / \mathrm{mL}$ increase in plasma PIIINP levels, the likelihood of having F $\geq 2$ fibrosis stage increased by $\sim 14$-fold even after adjusting for age, sex, BMI, HOMAestimated insulin resistance, NAS score and the PNPLA3 rs738409 variant.

Establishing and monitoring NAFLD disease severity is a major challenge for pediatricians caring for the growing number of children/adolescents with NAFLD. Therefore, there is a 
great need to develop non-invasive, easy-to-use, and cost-effective methods for replacing liver biopsy for staging the severity of liver fibrosis in NAFLD, which has been shown to be the strongest predictor of overall and liver-related mortality in adults with NAFLD. ${ }^{6-10}$ During the last decade, new circulating biomarkers have been proposed and evaluated for this purpose. Most of these biomarkers are biological components involved in the different steps of hepatic fibrogenesis, ECM remodeling proteins or fragments, such as PIIINP. ${ }^{12}$ PIIINP is proportionally detached from procollagen type III and released into the blood and urine during collagen cleavage. ${ }^{26,27}$ Therefore, an increase in PIIINP could be the result of increased synthesis and/or degradation of collagen. Thus, circulating levels of PIIINP have emerged as a key non-invasive biomarker of hepatic fibrogenesis. ${ }^{28,29}$

In some observational studies, plasma PIIINP levels have been shown to be strongly associated with liver fibrosis in adults with various chronic liver diseases (including NAFLD). ${ }^{15,30,31}$ Hamza et al. also reported that mean serum levels of PIIINP in children with NAFLD (assessed by ultrasonography) were higher than those in control children. ${ }^{16}$ Recently, Nielsen et al. ${ }^{32}$ have developed the PRO-C3 assay (i.e., a neo-epitope specific serum ELISA for type III procollagen) that could accurately predict advanced fibrosis in adults with NAFLD, when this serum biomarker is included into an algorithm with other clinical parameters, including the presence of diabetes and platelet count (i.e., the ADAPT score) ${ }^{33}$ In fact, this algorithm-derived score showed an AUROC of 0.86 (95\% Cl 0.79-0.91) for advanced liver fibrosis in adults with NAFLD. ${ }^{33}$ In line with this finding, in an international cohort of 449 adult patients with biopsy diagnosed NAFLD, Boyle et al. ${ }^{34}$ recently reported that plasma PRO-C3 levels correlated with the histologic stage of liver fibrosis, exhibiting similar performance to current fibrosis scores, such as FIB4 for detection of $\mathrm{F} \geq 3$ fibrosis. However, when PRO-C3 level was included into an algorithm with age, BMI and platelet count (i.e., the ABC3D score), this algorithm-derived score exhibited substantially improved accuracy (AUROC 0.83; 95\% Cl 0.79-0.87) and outperformed FIB4 and other similar diagnostic panels. ${ }^{34}$ 
In our study, we found that mean plasma PIIINP levels were $5.5 \mathrm{ng} / \mathrm{mL}$ in children with no $\mathrm{NASH} /$ borderline $\mathrm{NASH}$ and $8.6 \mathrm{ng} / \mathrm{mL}$ in those with $\mathrm{NASH}$, respectively. Moreover, we also showed that a cut-off of plasma PIIINP $>7.60 \mathrm{ng} / \mathrm{mL}$ was able to discriminate NASH from no$\mathrm{NASH} /$ borderline $\mathrm{NASH}$ with a sensitivity of $62 \%$, and specificity of $91 \%$. Notably, and most interestingly, we found that plasma PIIINP levels showed excellent diagnostic performance for predicting liver fibrosis stage. Indeed, AUROCs for plasma PIIINP levels for predicting either $\mathrm{F} \geq 2$ fibrosis or $\mathrm{F} 3$ fibrosis were $0.921(95 \% \mathrm{Cl} 0.87-0.97)$ and $0.993(95 \% \mathrm{Cl} 0.98-1.0)$, respectively. In particular, a cut-off of plasma PIIINP $>8.89 \mathrm{ng} / \mathrm{mL}$ yielded $84 \%$ sensitivity, 94\% specificity, 95\% PPV and 79\% NPV for predicting F $\geq 2$ fibrosis, whereas a cut-off of plasma PIIINP >13.2 ng/mL yielded $100 \%$ sensitivity, $98 \%$ specificity, $70 \%$ PPV and $100 \%$ NPV for predicting F3 fibrosis.

Our results suggest that the performance of PIIINP levels for staging the severity of liver fibrosis appears to be better for children than that reported previously in adults. Indeed, Tanwar et al. ${ }^{15}$ found that in 172 adult patients with biopsy-proven NAFLD (recruited from two specialist liver centers in the United Kingdom), the AUROC for plasma PIIINP levels in discriminating between $\mathrm{NASH}$ and simple steatosis was $0.77-0.82$ in patients with $\mathrm{F} 0-2$ fibrosis and 0.82-0.84 in patients with F0-3 fibrosis, respectively. It is unclear why the diagnostic accuracy of plasma PIIINP is much better in our cohort of children than in adults. However, it reasonable to hypothesize that compared to adults, children/adolescents with NAFLD are affected by far fewer of the comorbid extra-hepatic conditions that are known to affect circulating levels of PIIINP.

Our results appear to be consistent with those previously reported with the use of ELF test for predicting liver fibrosis stage in a small series of children with biopsy-proven NAFLD. ${ }^{13}$ In that study Nobili et al. found that the AUROCs/best possible ELF test cut-off values for the prediction of significant $(\mathrm{F} \geq 2)$, or advanced $(\mathrm{F} \geq 3)$ liver fibrosis were $0.98 / 10.18$ and 0.99/10.51, respectively. ${ }^{13}$ Unfortunately, the ELF test was not available in our cohort. 
Consequently, although there is no currently a head-to-head comparison in the diagnostic performances of plasma PIIINP levels and the ELF test in a pediatric population with NAFLD, we believe that the PIIINP measurement in this context might facilitate cost saving and its wider use in clinical practice when compared with the use of the ELF test that is a proprietary test offered by a single manufacturer. Indeed, the commercially available ELISA kits for measuring plasma PIIINP levels are much less expensive than the ELF test, and are also more easily available on all commercial laboratory auto-analyzers.

Interestingly, our results also show that both APRI and FIB-4 scores had poor performance for predicting the more advanced stages of liver fibrosis compared to plasma PIIINP levels. This finding is consistent with that reported by Mansoor et al..$^{35}$ showing that four noninvasive liver fibrosis scores developed in adults (i.e., the AST-to-ALT ratio, FIB-4, APRI and NAFLD fibrosis scores) had poor performance in diagnosing significant liver fibrosis in a sample of 92 United States children/adolescents with biopsy-confirmed NAFLD. Therefore, whereas these non-invasive liver fibrosis scores show promising results in adults with NAFLD ${ }^{10,24}$, the same cannot be said about children with NAFLD, based on our findings and those of Mansoor's study. ${ }^{35}$ The most important limitation for the clinical use of FIB-4 score in children is the weighting given to age in the formula. Consequently, the FIB 4 cut-offs proposed for ruling in/ruling out advanced liver fibrosis in adults are possibly not suitable for children. ${ }^{25,36}$ Indeed, in an European cohort of 634 patients with biopsy-proven NAFLD who attended specialist fatty liver clinics, McPherson et al. reported that the FIB-4 score performed poorly for diagnosing advanced liver fibrosis in patients aged $<35$ years (AUROC $0.60,95 \% \mathrm{Cl} 0.40-0.81$ ), suggesting that clinicians should use alternative means of noninvasive diagnosis of liver fibrosis in young people. ${ }^{37}$

The present study has some important strengths, but also some limitations that should be mentioned. For example, our study has a relatively large sample size, consecutive enrollment of subjects, completeness of the database, and the use of liver biopsy for diagnosing and 
staging NAFLD disease severity. Additionally, we believe that the examination of children/adolescents with NAFLD, who are affected by fewer comorbidities (than adults), offers the opportunity to explore the associations between plasma PIIINP levels and the severity of NAFLD histology without the influence of other potential confounding factors. Conversely, the absence of a control population and the small number of cases with F3 fibrosis $(n=7)$ are the major limitations of our study. However, advanced liver fibrosis is rare in children with NAFLD and it would be unethical to subject children without NAFLD to a general anaesthetic and liver biopsy, in order to confirm that a control group of children did not have NAFLD. Finally, the lack of a validation cohort cautions against the generalizability of our findings to other pediatric groups. Further research is needed to validate our optimal cutoff of plasma PIIINP for detecting significant liver fibrosis in larger independent series, and it is also advisable to compare our data obtained with plasma PIIINP with those obtainable by using plasma PRO-C3 in future pediatric studies. Moreover, since we showed that plasma PIIINP levels were a strong predictor of liver fibrosis stage, it is plausible that this biomarker could serve also for monitoring pediatric NAFLD during follow-up, thus avoiding any need for a repeat liver biopsy.

In conclusion, the results of our study show that in contrast to APRI and FIB-4 scores, plasma PIIINP levels represent a promising, non-invasive biomarker for diagnosing liver fibrosis stage in children/adolescents with NAFLD. However, these results will require further validation in other cohorts of children/adolescents with biopsy-proven NAFLD.

\section{REFERENCES}

1. Panera N, Barbaro B, Della Corte C, Mosca A, Nobili V, Alisi A. A review of the pathogenic and therapeutic role of nutrition in pediatric nonalcoholic fatty liver disease. Nutr Res 2018;58:1-16. 
2. Mann JP, Valenti L, Scorletti E, Byrne CD, Nobili V. Nonalcoholic fatty liver disease in children. Semin Liver Dis 2018;38:1-13.

3. Anderson EL, Howe LD, Jones HE, Higgins JP, Lawlor DA, Fraser A. The prevalence of non-alcoholic fatty liver disease in children and adolescents: a systematic review and meta-analysis. PLoS One 2015;10:e0140908.

4. Nobili V, Alisi A, Newton KP, Schwimmer JB. Comparison of the phenotype and approach to pediatric vs adult patients with nonalcoholic fatty liver disease. Gastroenterology 2016;150:1798-1810.

5. Goyal NP, Schwimmer BJ. The progression and natural history of pediatric nonalcoholic fatty liver disease. Clin. Liver Dis 2016;20:325-338.

6. Angulo $\mathrm{P}$, Kleiner DE, Dam-Larsen $\mathrm{S}$, et al. Liver fibrosis, but no other histologic features, is associated with long-term outcomes of patients with nonalcoholic fatty liver disease. Gastroenterology 2015;149:389-397.e10.

7. Ekstedt $M$, Hagström $H$, Nasr $P$, et al. Fibrosis stage is the strongest predictor for disease-specific mortality in NAFLD after up to 33 years of follow-up. Hepatology 2015;61:1547-1554.

8. Younossi ZM, Stepanova M, Rafiq N, et al. Pathologic criteria for nonalcoholic steatohepatitis: interprotocol agreement and ability to predict liver-related mortality. Hepatology 2011;53:1874-1882. 
9. Hagström H, Nasr P, Ekstedt $\mathrm{M}$, et al. Fibrosis stage but not NASH predicts mortality and time to development of severe liver disease in biopsy-proven NAFLD. J Hepatol $2017 ; 67: 1265-1273$

10.Byrne CD, Patel J, Scorletti E, Targher G. Tests for diagnosing and monitoring nonalcoholic fatty liver disease in adults. BMJ 2018;362:k2734.

11.Vilar-Gomez E, Chalasani N. Non-invasive assessment of nonalcoholic fatty liver disease: clinical prediction rules and blood-based biomarkers. $\quad J$ Hepatol 2018;68:305-315.

12. Guha IN, Parkes J, Roderick P, et al. Noninvasive markers of fibrosis in nonalcoholic fatty liver disease: validating the European Liver Fibrosis Panel and exploring simple markers. Hepatology 2008;47:455-460.

13. Nobili V, Parkes J, Bottazzo G, et al. Performance of ELF serum markers in predicting fibrosis stage in pediatric non-alcoholic fatty liver disease. Gastroenterology 2009;136:160-167.

14.Frei A, Zimmermann A, Weigand K. The N-terminal propeptide of collagen type III in serum reflects activity and degree of fibrosis in patients with chronic liver disease. Hepatology 1984;4:830-834. 
15. Tanwar S, Trembling PM, Guha IN, et al. Validation of terminal peptide of procollagen III for the detection and assessment of nonalcoholic steatohepatitis in patients with nonalcoholic fatty liver disease. Hepatology 2013;57:103-111.

16. Hamza RT, Ahmed AY, Rezk DG, Hamed Al. Dietary fructose intake in obese children and adolescents: relation to procollagen type III N-terminal peptide (P3NP) and nonalcoholic fatty liver disease. J Pediatr Endocrinol Metab 2016; 29:1345-1352.

17. Vajro $P$, Lenta $S$, Socha $P$, et al. Diagnosis of nonalcoholic fatty liver disease in children and adolescents: position paper of the ESPGHAN Hepatology Committee. J Pediatr Gastroenterol Nutr 2012;54:700-713.

18.Wijnhoven TM, van Raaij JM, Spinelli A, et al. WHO European Childhood Obesity Surveillance Initiative: body mass index and level of overweight among 6-9-year-old children from school year 2007/2008 to school year 2009/2010. BMC Public Health 2014:806.

19.Farpour-Lambert NJ, Baker JL, Hassapidou M, et al. Childhood obesity is a chronic disease demanding specific health care-a position statement from the Childhood Obesity Task Force (COTF) of the European Association for the Study of Obesity (EASO). Obes Facts 2015;8:342-349.

20.Conwell LS, Trost SG, Brown WJ, Batch JA. Indexes of insulin resistance and secretion in obese children and adolescents: a validation study. Diabetes Care 2004;27:314-319. 
21.Kleiner DE, Brunt EM, Van Natta M, et al; Nonalcoholic Steatohepatitis Clinical Research Network. Design and validation of a histological scoring system for nonalcoholic fatty liver disease. Hepatology 2005;41:1313-1321.

22. Brunt EM, Kleiner DE, Wilson LA, NASH Clinical Research Network (CRN). Nonalcoholic fatty liver disease (NAFLD) activity score and the histopathologic diagnosis in NAFLD: distinct clinic pathologic meanings. Hepatology 2011;53:810-820.

23. Santiago-Rolón A, Purcell D, Rosado K, Toro DH. A comparison of Brunt's criteria, the non-alcoholic fatty liver disease activity score (NAS), and a proposed NAS scoring that includes fibrosis in non-alcoholic fatty liver disease staging. $P$ R Health Sci $J$ 2015;34:189-194.

24.Castera L, Friedrich-Rust M, Loomba R. Noninvasive assessment of liver disease in patients with nonalcoholic fatty liver disease. Gastroenterology 2019;156:1264-1281.

25. Mansoor S, Collyer E, Alkhouri N. A comprehensive review of noninvasive liver fibrosis tests in pediatric nonalcoholic fatty liver disease. Curr Gastroenterol Rep 2015;17:23.

26.Prockop DJ, Kivirikko KI, Tuderman L, Guzman NA. The biosynthesis of collagen and its disorders (first of two parts). N Engl J Med 1979;301:13-23.

27. Niemelä O, Risteli L, Parkkinen J, Risteli J. Purification and characterization of the Nterminal propeptide of human type III procollagen. Biochem J 1985;232:145-150. 
28. Sugimoto $M$, Saiki $H$, Tamai $A$, et al. Ventricular fibrogenesis activity assessed by serum levels of procollagen type III N-terminal amino peptide during the staged Fontan procedure. J Thorac Cardiovasc Surg 2016;151:1518-1526.

29. Ghoul BE, Squalli T, Servais A, et al. Urinary procollagen III aminoterminal propeptide (PIIINP): a Fibrotest for the nephrologist. Clin J Am Soc Nephrol 2010;5:205-210.

30.Remmel T, Remmel H, Salupere V. Aminoterminal propeptide of type III procollagen and hyaluronan in patients with primary biliary cirrhosis: markers of fibrosis in primary biliary cirrhosis. J Gastroenterol Hepatol 1996;11:1016-1020.

31. Guéchot J, Laudat A, Loria A, Serfaty L, Poupon R, Giboudeau J. Diagnostic accuracy of hyaluronan and type III procollagen amino-terminal peptide serum assays as markers of liver fibrosis in chronic viral hepatitis $\mathrm{C}$ evaluated by ROC curve analysis. Clin Chem 1996;42:558-563.

32.Nielsen MJ, Nedergaard AF, Sun S, et al. The neo-epitope specific PRO-C3 ELISA measures true formation of type III collagen associated with liver and muscle parameters. Am J TransI Res 2013;5:303-315.

33. Daniels SJ, Leeming DJ, Eslam M, et al. ADAPT: an algorithm incorporating PRO-C3 accurately identifies patients with NAFLD and advanced fibrosis. Hepatology 2019;69:1075-1086.

34.Boyle M, Tiniakos D, Schattenberg JM, et al. Performance of the PRO-C3 collagen neo-epitope biomarker in non-alcoholic fatty liver disease. JHEP Reports 2019; in press, https://doi.org/10.1016/j.jhepr.2019.06.004. 
35. Mansoor S, Yerian L, Kohli R, et al. The evaluation of hepatic fibrosis scores in children with nonalcoholic fatty liver disease. Dig Dis Sci 2015;60:1440-1447.

36. Nobili V, Alisi A, Valenti L, Miele L, Feldstein AE, Alkhouri N. NAFLD in children: new genes, new diagnostic modalities and new drugs. Nat Rev Gastroenterol Hepatol 2019 Jul 5. doi: 10.1038/s41575-019-0169-z [Epub ahead of print].

37. McPherson S, Hardy T, Dufour JF, et al. Age as a confounding factor for the accurate non-invasive diagnosis of advanced NAFLD fibrosis. Am J Gastroenterol $2017 ; 112: 740-751$. 


\section{FIGURE LEGENDS}

Figure 1. Plasma levels of PIIINP in children with biopsy diagnosed NAFLD.

Boxplots show plasma PIIINP levels in children (A) with definite NASH compared with either those with borderline NASH and/or not-NASH considered together, or in children (B) with not$\mathrm{NASH}$ and borderline NASH considered separately). In panel $\mathrm{B}$, all inter-group differences in plasma PIIINP levels were statistically significant $(p<0.0001$ by Bonferroni's multiple comparison tests).

Figure 2. Stratification of plasma levels of PIIINP in children according to histologic features of NAFLD.

Boxplots show plasma PIIINP levels in children stratified by histologic features of NAFLD, including (A) degree of fibrosis, (B) ballooning, (C) lobular inflammation, (D) portal inflammation, and $(E)$ steatosis; and $(F)$ based on NAS. In panels $A$ to $D$, all inter-group differences in plasma PIIINP levels were statistically significant $(p<0.0001$ by Bonferroni's multiple comparison tests). In panel E, significant inter-group differences in plasma PIIINP levels were found only for the comparison of hepatic steatosis $<5 \%$ vs. each other grade of steatosis $(p<0.005-0.001$ by Bonferroni's multiple comparison tests). In panel $\mathrm{F}$, significant inter-group differences in plasma PIIINP levels were found only for the comparison of NAS 02 vs. each other NAS $\geq 3$ ( $p<0.05-0.0001$ by Bonferroni's multiple comparison tests).

Figure 3. ROC curves for definite NASH and different liver fibrosis thresholds.

Diagnostic performances of plasma PIIINP levels, as assessed by the AUROC, for discriminating (A) NASH, (B) significant fibrosis $(F \geq 2)$ and $(C)$ advanced fibrosis $(F \geq 3)$ in children with biopsy-proven NAFLD. 
Figure 4. Comparison of ROC curves for definite NASH and different liver fibrosis thresholds.

Diagnostic performances of plasma PIIINP levels, FIB-4 and APRI scores for discriminating (A) NASH, (B) significant fibrosis $(F \geq 2)$ and $(C)$ advanced fibrosis $(F \geq 3)$ in children with biopsy-proven NAFLD. 